\title{
Notes on the Reproductive Biology of the Big-eyed Ratsnake, Ptyas dhumnades (Cantor, 1842) in Taiwan
}

\author{
Simon Dieckmann ${ }^{1}$, Gerrut Norval2 ${ }^{2 *}$, and Jean-Jay $\mathrm{Mao}^{3}$ \\ ${ }^{1}$ No. 430 Huacheng $5^{\text {th }}$ Str., Ji-an Township, Hualien County, 973, Taiwan, R.O.C. (gujonaan@yahoo.de) \\ ${ }^{2}$ Applied Behavioural Ecology \& Ecosystem Research Unit, Department of Environmental Sciences, UNISA, \\ Private Bag X6, Florida, 1710, Republic of South Africa (gnorval@gmail.com) \\ ${ }^{3}$ Department of Forestry and Natural Resources, National Ilan University. No. 1, Sec. 1, Shen-Lung Rd., Ilan, 260, \\ Taiwan, R.O.C. (jjmao@niu.edu.tw) \\ *corresponding author
}

$\mathrm{T}$

The Big-eyed Ratsnake (Ptyas dhumnades [Cantor, 1842], formerly classified as Zaocys dhumnades; Fig. 1) (David and Vogel 1996) has a natural distribution that extends through parts of Vietnam, central and southern China, and Taiwan (Kuntz 1963, Pope 1935, Orlov et al. 2000). In Taiwan, P. dhumnades is fairly common and tends to inhabit grassy and wooded foothills and some agricultural areas (Kuntz 1963, Lue et al. 2002). Herein we describe a clutch of eggs from $P$. dhumnades in Taiwan.

On 12 June 2014, a female Big-eyed Ratsnake was collected from the wild in the foothill region of Huacheng, Hualien County, eastern Taiwan (the exact locality was not recorded). The snake, which had a total length of $118 \mathrm{~cm}$, was

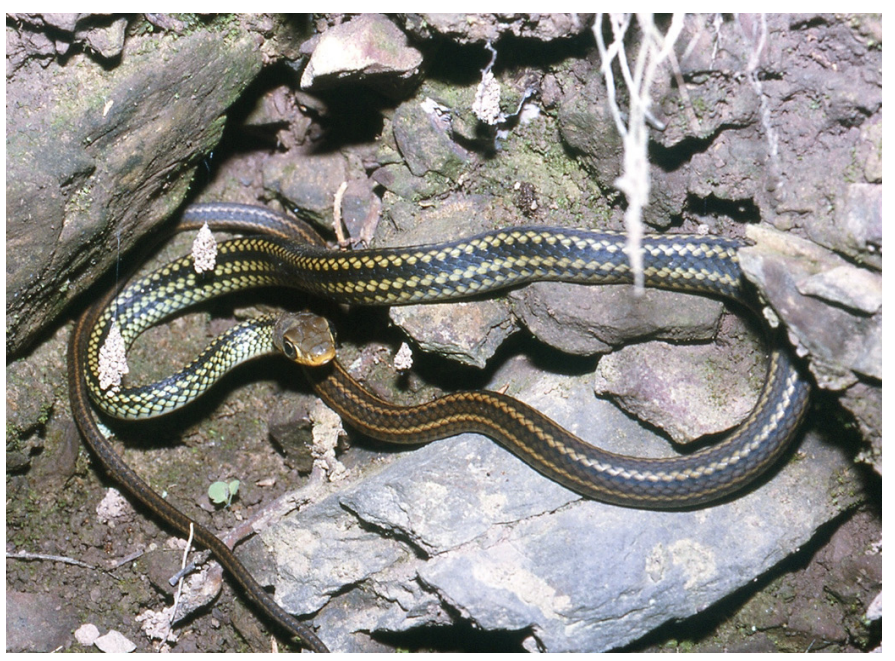

Fig. 1. The Big-eyed Ratsnake (Ptyas dhumnades) is relatively common in Taiwan, but rarely seen due to their secretive nature and agility. This individual was photographed in the Dajioushi Experimental Forest in Yilan County, northeastern Taiwan, at an elevation of $500 \mathrm{~m}$. Photograph by Jean-Jay Mao. kept in a cage that had a length, width, and height of 120,60 , and $60 \mathrm{~cm}$, respectively. The cage contained a dry and a moist shelter, branches for climbing, and a large water bowl. On the morning of 17 June, we found a clutch of eggs in the water on top of the moist shelter (Fig. 2). The eggs were bound to each other and had a combined weight of $69 \mathrm{~g}$. The eggs were placed inside an incubator in which the temperature varied from 27 to $30^{\circ} \mathrm{C}$ and the humidity was maintained at $90-95$ $\%$. The relative clutch mass (RCM) was calculated using the formula: total clutch weight / maternal post-oviposition body weight (Shine 1980, Shine 1992). On 2-3 August, after an

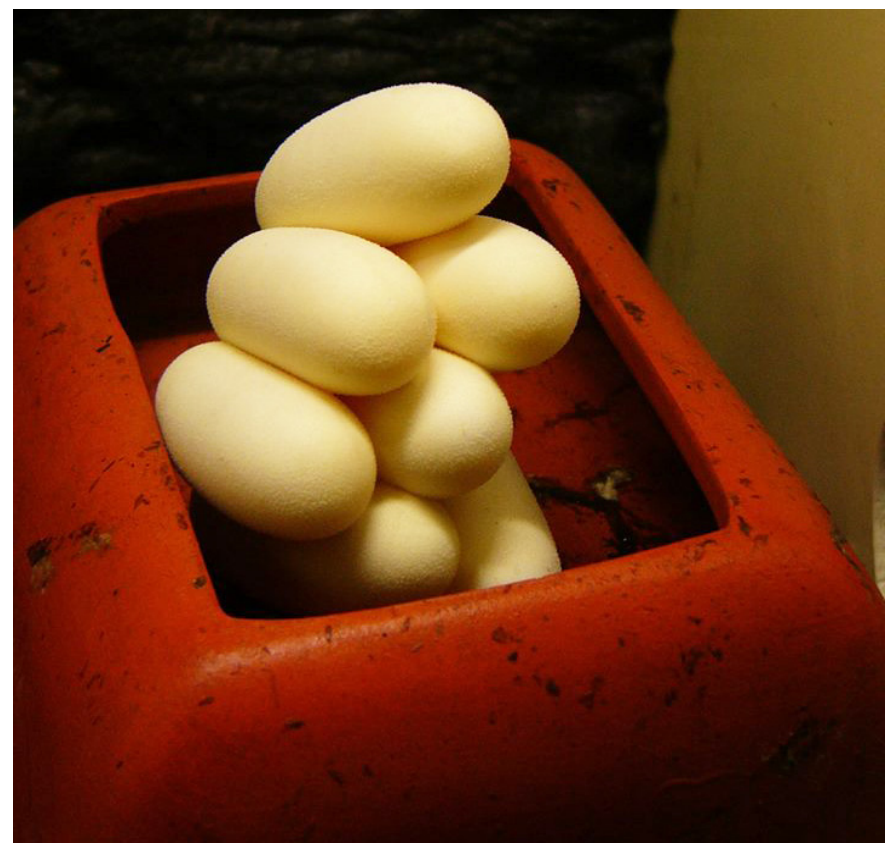

Fig. 2. Big-eyed Ratsnake (Ptyas dhumnades) eggs were found in the water on top of the moist shelter. Photograph by Simon Dieckmann. 
incubation period of 48 days, five of the eggs hatched (Fig. 3). The two eggs that failed to hatch were rotten. To avoid injury to the hatchlings, no attempts were made to sex them, but total lengths (measured with a ruler to the nearest $\mathrm{cm}$ ) were all $30 \mathrm{~cm}$, and body weights ranged from 9 to $10 \mathrm{~g}$ (mean \pm $\mathrm{SD}=9.8 \pm 0.5)$. The hatchlings were housed independently in small plastic animal cages (length $\mathrm{x}$ width $\mathrm{x}$ height $=45 \mathrm{x}$ $25 \times 25 \mathrm{~cm}$ ) with paper towels as a substrate; each cage was provided with a water bowl and a shelter. On 15 September, the female and all of the hatchlings were released back into the wild in the vicinity of where the female was collected.

An understanding of the natural history of a species is essential for successful conservation and management programs (Greene 1994, Bury 2006), and a crucial part of the natural history of a species is its reproductive biology. Although information from animals in captivity should be interpreted with caution, it can be incorporated with data collected from the wild to develop a better understanding of the natural his-

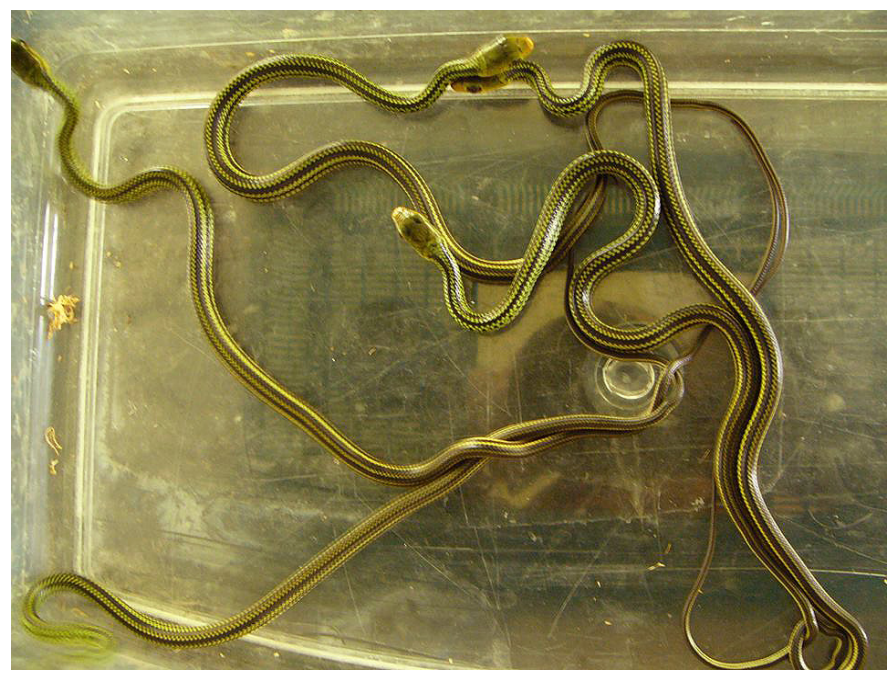

Fig. 3. Big-eyed Ratsnake (Ptyas dhumnades) hatchlings. Photograph by Simon Dieckmann. tory of a species. In our literature review, we found some references to some aspects of the reproductive biology of $P$. dhumnades (Table 1). The eggs described herein resembled those reported by Pope (1935), who stated that in $P$. dhumnades from China, the eggs are attached to each other when laid and shells have a rough surface. The eggs were similar in size to those reported by other authors, but were lighter than the lowest clutch weight described by Ji et al. (2000). Still, the RCM was higher than the mean RCM described by Ji et al. (2000). The female from our study was within the size range described by Ji et al. (2000), so the relatively high RCM is a result of a low body weight of the female. Since the snake was in captivity less than a week prior to oviposition, we concluded that the low body weight and clutch mass were most likely a result of natural conditions. The incubation period in our study is longer than the one month reported by $\mathrm{Tu}$ (2004), and is likely a result of lower incubation temperatures. No hatchling size descriptions were found, so we can not make any compari-

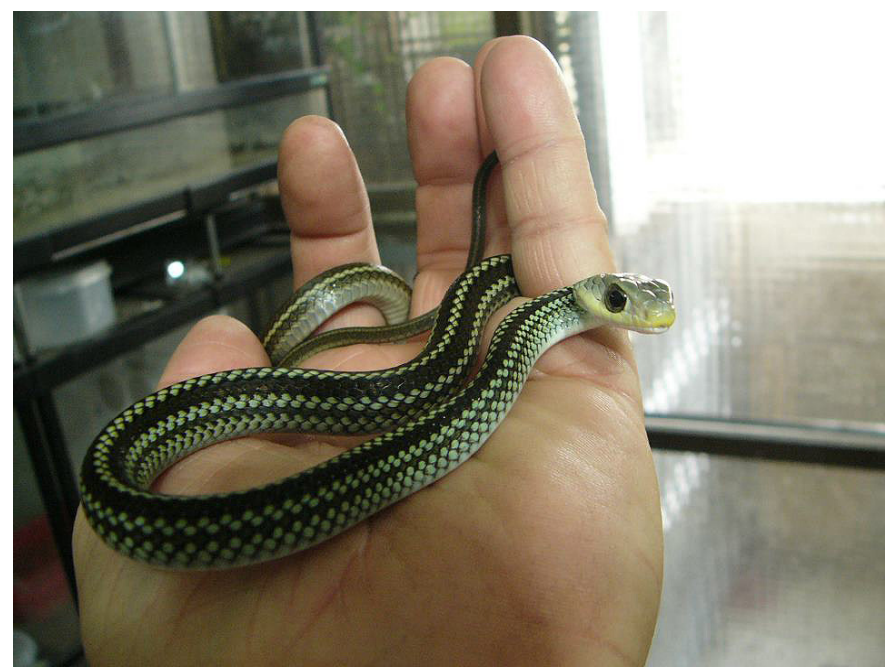

Fig. 4. A captive Big-eyed Ratsnake (Ptyas dhumnades) exhibiting the coloration characteristic of this species. Photograph by Simon Dieckmann.

Table 1. A summary of references to clutch sizes, egg dimensions, clutch masses, and relative clutch masses (RCM) of Big-eyed Ratsnakes (Ptyas dhumnades). ${ }^{1}$ reported as Zaocys dhumnades dhumnades, ${ }^{2}$ reported as Zaocys dhumnades montanus (both subspecies currently regarded as synonyms for Ptyas dhumnades).

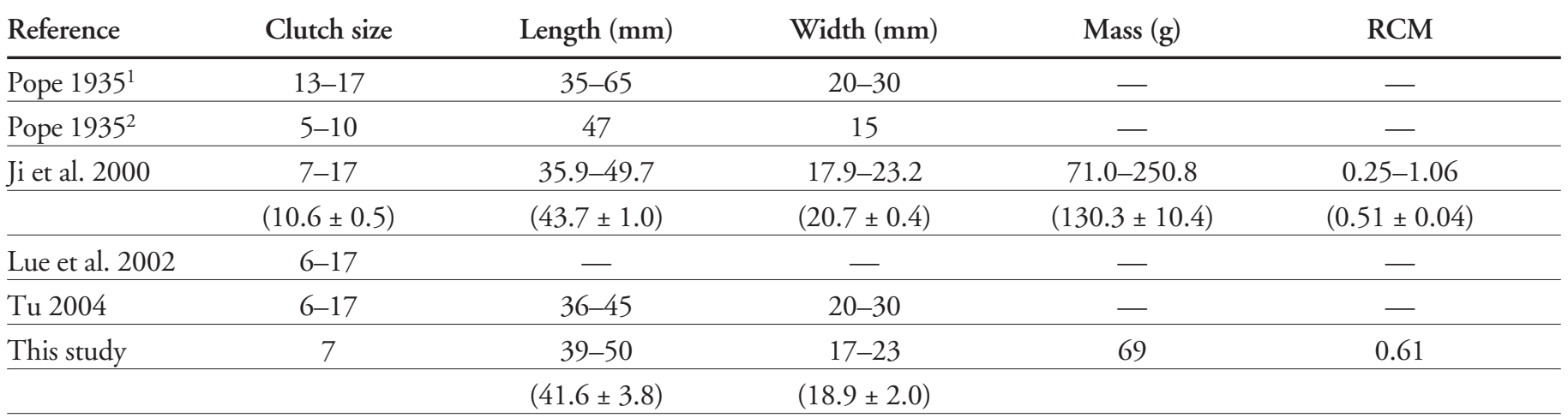


sons. The coloration of the hatchlings was similar to that of the adults (Fig. 4). Also, Pope (1935) and Ji et al. (2000) noted reproduction in June and July, so the oviposition period of the female described herein is not out of the ordinary. Based on the available information, the reproductive biology of $P$. dhumnades appears to be similar throughout its distribution. To our knowledge, this is the first reported description of relative clutch mass in a Big-eyed Ratsnake from Taiwan.

\section{Literature Cited}

Bury, B.R. 2006. Natural history, field ecology, conservation biology and wildlife management: Time to connect the dots. Herpetological Conservation and Biology 1:56-61.

David, P. and G. Vogel. 1996. The Snakes of Sumatra. An Annotated Checklist and Key with Natural History Notes. Edition Chimaira, Frankfurt am Main, Germany.
Greene, H.W. 1994. Systematics and natural history, foundations for understanding and conserving biodiversity. American Zoologist 34:48-56.

Ji, X., P.Y. Sun, X.F. Xu, and W.G. Du. 2000. Relationships among body size, clutch size, and egg size in five species of oviparous colubrid snakes from Zhoushan Island, Zhejiang, China. Acta Zoologica Sinica 46:138-145 (in Chinese).

Kuntz, R.E. 1963. Snakes of Taiwan. Quarterly Journal of Taiwan Museum 16:11-12.

Lue, K.Y., M.C. Tu, and G.S. Shang. 2002. The Transition Kingdom - Guidebook of Amphibians and Reptiles of Taiwan. SWAN, Taipei (in Chinese).

Orlov N.L., R.W. Murphy, and T.J. Papenfuss. 2000. List of snakes of Tam-Dao mountain ridge (Tonkin, Vietnam). Russian Journal of Herpetology 7:69-80.

Pope, C.H. 1935. The reptiles of China: Turtles, crocodilians, snakes, lizards. Series: Natural History of Central Asia. American Museum of Natural History 10:1-604.

Shine, R. 1980. "Costs" of reproduction in reptiles. Oecologia 46:92-100.

Shine, R. 1992. Relative clutch mass and body shape in lizards and snakes: Is reproductive investment constrained or optimized? Evolution 46:828-833.

Tu, M.C. 2004. Big Surprise of Snakes. Yuan-Liou Publishing Co. Ltd., Taipei (in Chinese). 